\title{
EXPLORANDO RELACIONES ENTRE LA RESOLUCIÓN DE PROBLEMAS DE FÍSICAY MATEMÁTICA
}

\section{EXPLORING RELATIONSHIPS BETWEEN SOLVING PROBLEMS IN PHYSICS AND MATHEMATICS}

\section{Luis Fernando Mariño', \\ César Augusto Hernández Suárez²}

\section{Raúl Prada Núñez ${ }^{3}$}

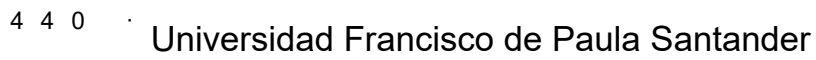

\section{RESUMEN}

Las caracterizaciones acerca de la resolución de problemas de física y matemática generalmente se han realizado por vías diferentes. En contraste, estudios conjuntos de estos dos constructos han sido poco abordados.

1 Doctor en Educación Matemática por la Universidad Antonio Nariño (Colombia). Magister en Educación Matemática. Licenciado en Matemáticas y Computación. Profesor Departamento de Matemáticas y Estadística de la Universidad Francisco de Paula Santander (Cúcuta). Correo. fernandoml@ufps.edu.co. Orcid: https://orcid. org/0000-0002-3438-6963

2 Magister en Enseñanza de las Ciencias mención Matemática por la Universidad Nacional Experimental del Táchira (Venezuela). Docente investigador de la Universidad Francisco de Paula Santander. Correo electrónico: cesaraugusto@ufps.edu.co. Orcid: https://orcid.org/00000002-7974-5560

3 Magíster en Ingeniería de Análisis de Datos, Mejora de Procesos y Toma de Decisiones por la Universidad Politécnica de Valencia (España). Docente investigador de la Universidad Francisco de Paula Santander. Correo electrónico: raulprada@ufps.edu.co. Orcid: https://orcid. org/0000-0001-6145-1786
Este reporte, muestra los resultados de una investigación cualitativa, desde un estudio de caso que responde a la pregunta: ¿cómo son las relaciones entre la resolución de problemas de física y matemática que involucran circuitos y sistemas de ecuaciones lineales, manifestadas por un grupo de estudiantes de ingeniería de sistemas? Como fuentes de recolección de información se diseñaron e implementaron seis secuencias de aprendizajes. El análisis de datos se realizó siguiendo los procesos simultáneos de codificación abierta, axial y selectiva. Los hallazgos muestran como los estudiantes utilizan el conocimiento que tiene a su disposición, descubren y construyen relaciones matemáticas formalizando leyes físicas, además de generalizar y utilizar métodos para resolver sistemas de ecuaciones lineales. Los resultados evidencian una relación clara de inclusión de 
las estrategias para resolver problemas de matemáticas entre las estrategias para resolver problemas de física, caracterizadas por un flujo de trabajo simultaneo, cíclico y permanente sobre el problema.

\section{PALABRAS CLAVE}

Resolución de problemas de física, Resolución de problemas de matemáticas, sistemas de ecuaciones lineales, estudio de caso.

\section{ABSTRACT}

Characterizations about solving problems in physics and mathematics have generally been done in different ways. In contrast, joint studies of these two constructs have been little addressed. This report shows the results of a qualitative research, from a case study that answers the question: how are the relationships between the resolution of physics and mathematics problems involving circuits and systems of linear equations, manifested by a group of systems engineering students? Six learning sequences were designed and implemented as sources of information collection. Data analysis was performed following the simultaneous processes of open, axial, and selective coding. The findings show how students use the knowledge at their disposal, discover and build mathematical relationships by formalizing physical laws, as well as generalizing and using methods to solve systems of linear equations. The results show a clear relationship of inclusion of strategies to solve mathematical problems among strategies to solve physics problems, characterized by a simultaneous, cyclical, and permanent workflow on the problem.

KEY WORDS: Solving physics problems, solving math problems, linear equation systems, case study.

\section{INTRODUCCIÓN}

Un obstáculo crucial en la formación inicial del ingeniero es la resolución de problemas ya sea de física o matemática. Posiblemente, en su formación en los niveles precedentes, los estudiantes acumularon una serie de conocimientos y habilidades en matemáticas, pero al momento de resolver problemas de física, se evidencian serias dificultades (Scanlon, 1993; Redish, 2006). Desde la física estas dificultades pueden estar relacionadas con la construcción del modelo que representa la situación y el conocimiento base del solucionador (Scanlon, 1993). Desde la matemática por su parte, probablemente estas falencias se relacionen con procesos mentales, como la generalización, la prueba, además de técnicas y habilidades en procedimientos.

La matemática es fundamental en la resolución de problemas de física (Redish, 2006; Bing y Redish, 2009). Resolver problemas de física sin el uso del conocimiento matemático, parece poco probable e inevitable (De Ataíde y Greca, 2012; Al-Omari y Miqdadi, 2014). La matemática proporciona un lenguaje que permite expresar, representar relaciones y leyes físicas, que van desde el uso de una variedad de letras y símbolos para representar variables y constantes (Redish E. , 2006), hasta la formalización, solución y verificación de la solución del problema en el dominio de cada una de estas áreas. Como lo reitera Quale (2010), quien afirma que hay dos formas de estudiar fenómenos físicos, primero el juicio y la observación y segundo el pensamiento matemático.

En las últimas décadas, la resolución de problemas tanto de matemáticas como de física ha despertado el interés en comunidades científicas y académicas. Este interés se centra generalmente en intentar conocer los procesos cognitivos, las estructuras del conocimiento $\mathrm{y}$ las estrategias para resolver problemas. 
Tradicionalmente, estos estudios han sido abordados desde una diversidad de enfoques y perspectivas en diferentes dominios, por vías independientes y no de manera conjunta.

Desde la matemática y lo cognitivo, investigadores clásicos y contemporáneos han propuesto procesos, fases o etapas para resolver problemas. Polya $(1945 ; 1981)$ formuló una serie de cuatro fases que denominó, entender el problema, idear un plan, llevar a cabo el plan y mirar hacia atrás. Mason, Burton y Stacey (2010), plantearon las fases en entrada, ataque y revisión. Mientras que Mayer (2010), caracterizó la resolución de problemas en dos grandes fases, que las denominó representación del problema y solución del problema.

Schoenfeld (2016), parece ampliar esta gama de caracterizaciones y determinó cinco dimensiones que intervienen directa, dinámica e interdependientemente: a) dimensión cognitiva (base de conocimientos), b) heurísticas, estrategias en la resolución de problemas, c) dimensión metacognitiva, monitoreo y control (auto-regulación), d) dimensión afectiva, creencias y afectos, y e) práctica matemática. Cada una de estas fases en las diversas caracterizaciones, están a su vez compuestas por una serie de etapas y acciones que debe poner en juego el solucionador del problema.

Desde la física y como producto de sus trabajos Gaigher, Rogan y Braun (2007) formularon una estrategia para resolver problemas y desarrollar comprensión conceptual. La estrategia se caracteriza por siete etapas: 1) dibujar un diagrama simple que represente el problema, 2) indicar los datos en el diagrama, 3) indentificar variables desconocidas, 4) analizar el problema en términos de principios y leyes fisicas, 5) escribir las ecuaciones relevantes, 6) sustituir y resolver, y 7) interpretar la respuesta numérica. Esta estrategia, coincide con la caractrización que se encuentra tradicionalmente en libros de texto: a) dibujar un boceto, b) definir cantidades conocidas y desconocidas, c) seleccionar ecuaciones, d) resolver ecuaciones; y e) comprobar la respuesta.

Por su parte, Jensen, Niss y Jankvist (2017), plantearon un modelo didáctico para la resolución de problemas de física, compuesto por tres etapas y dos procesos. El primer proceso es la formalización del problema, que consiste en un problema formulado ya sea física o matemáticamente. En este proceso, están inmersos los subprocesos de sistematización (elementos relevantes e irrelevantes de la situación) y traducción (expresar el problema en términos físicos o matemáticos, decidiendo variables, escribiendo ecuaciones, etc.). El segundo proceso, es la resolución del problema formalizado, ya sea en dominios de la matemática o en dominios de la física, para finalmente hallar la respuesta.

Desde un punto de vista más general, Greeno (1989), autor clásico propone un modelo bastante amplio para resolución de problemas científicos desde la comprensión conceptual. El modelo se caracteriza por cuatro dominios: 1) concreto (objetos físicos y eventos), 2) modelo (modelos de la realidad y abstracciones), 3) abstracto (conceptos, leyes y principios), y 4) simbólico (lenguaje y algebra).

Las investigaciones que hacen aportes en conocer, describir y contribuyen con estrategias para resolver problemas de matemáticas $\mathrm{y}$ física generalmente han sido abordadas desde enfoques, propósitos y caminos diferentes e independientes; aunque la realidad parece mostrar todo lo contrario. Otro elemento distintivo de estos trabajos es que el análisis de información surge de la lente del investigador y no de los datos manifestados por los participantes. En la enseñanza de la física, por ejemplo, existe una gran cantidad de trabajos acerca de 
las estrategias para resolver problemas entre expertos y novatos.

Ante esta situación, desde un contexto especifico y teniendo en cuenta que la resolución de problemas es una actividad intelectualmente exigente y de vital importancia para el ingeniero, tanto en su formación, como en su desempeño profesional, el estudio tuvo como propósito dar respuesta a la pregunta de investigación: ¿cómo son las relaciones entre la resolución de problemas de física y matemática que involucran circuitos y sistemas de ecuaciones lineales, manifestadas por un grupo de estudiantes de ingeniería de sistemas?

\section{METODOLOGÍA}

La pregunta de investigación, acerca de ¿cómo son las relaciones entre la resolución de problemas de física y matemática?, condujo a la adopción de un paradigma de investigación cualitativo siguiendo un estudio de caso.

El caso se caracteriza por dos elementos: primero, los participantes son estudiantes que inician su formación como ingenieros de sistemas y segundo, los resultados surgen de los procesos de análisis y codificación de los datos manifestados por los participantes. Esto lo reiteran Miles, Huberman y Saldaña (2018) quienes caracterizan un caso, como un fenómeno de algún tipo, que ocurre en un contexto determinado.

Participantes. Estuvo conformado por un grupo de 30 estudiantes, que tomaron un curso de Algebra Lineal, en el programa de Ingeniería de Sistemas de la Universidad Francisco de Paula Santander en la ciudad de Cúcuta (Colombia), durante el I semestre de 2021. El 76,7\% de las edades de los participantes oscilaban entre $17 \mathrm{y}$ 19 años, mientras que el $13,35 \%$ son mujeres.

Fuentes de datos empíricos. Como instrumentos para la recolectar los datos se diseñaron, e implementaron seis secuencias de aprendizaje. Estas secuencias, se caracterizaron por el uso simultaneo del método inductivo y deductivo en el aprendizaje, con la intención de que fuera el estudiante quien elaborara y construyera su propio conocimiento. Este tipo de estrategias es consecuente con el constructivismo, específicamente con el constructivismo social y radical, cuyo fundamento radica en que, la función de la cognición es adaptativa y sirve para organizar el mundo experiencial grupal e individualmente, no al descubrimiento de una verdad ontológica (Ernest, 2005; Von Glasersfeld, 2013).

EI trabajo empírico. Las tareas y actividades para dar respuesta a la pregunta de investigación siguieron un trabajo compuesto por tres etapas. Las etapas 1 y 2 corresponden a la implementación de las secuencias de aprendizaje, mientras que la etapa 3, hace referencia al proceso de análisis cualitativo de datos, que posibilitó la elaboración de las categorías centrales y su densidad de relaciones.

Es de vital importancia, resaltar que este trabajo fue realizado en tiempos de la pandemia originada por la COVID 2019, que afecta al mundo, incluso durante la elaboración del presente escrito. Lo que dio origen a una forma de comunicación sincrónica y asincrónica entre el profesor y los participantes, con la ayuda de la aplicación Google Meet. Los participantes realizaban sus actividades en hojas de papel, escaneaban en formato pdf y enviaban estos archivos al profesor investigador. Como resultado de este trabajo hay 54 documentos digitales en este formato.

Análisis de datos. La codificación y análisis de datos se realizó siguiendo los procesos de codificación abierta, axial y selectiva, propios de la Teoría Fundamentada (Charmaz, 2006; 2014; Corbin y Strauss, 2017). En la codificación abierta se seleccionan los primeros incidentes, en el sentido que fueron las manifestaciones 
escritas de los participantes que elaboraron un mejor trabajo. Surgen de allí los primeros códigos o conceptos indicadores, se denominan así, puesto que representan los datos reales.

De la codificación abierta surgieron una variedad de códigos iniciales, que se agruparon dando origen a las primeras categorías, a partir de un proceso de comparación y contrastación entre ellos. Luego el investigador se aleja un poco de los datos e inicia el proceso de codificación selectiva, con la intención de buscar conceptos de nivel superior, esto condujo finalmente a las categorías centrales. El análisis se realizó primero en la totalidad de un documento, luego otro y así sucesivamente, con el propósito de poder dar sentido a los datos o manifestaciones escritas de los participantes.

Como el propósito del trabajo estuvo centrado en la búsqueda de relaciones o diferencias entre la resolución de problemas de matemática y física, se presenta primero la codificación y análisis de cada uno de ellos y luego el trabajo conjunto. Aunque, la forma en que se presente parece ser lineal, no lo es, por el contrario, es un trabajo de ida y vuelta permanente sobre los datos.

La resolución de problemas matemáticos. Las tareas a resolver por los participantes en las secuencias de aprendizaje implementadas en la etapa 1, involucraron la resolución de sistemas de $m$ ecuaciones lineales con $n$ incógnitas o variables. Del proceso de análisis de las manifestaciones escritas de los estudiantes, surgieron los primeros códigos, a continuación, se describen algunos de ellos. Desde la Teoría Fundamentada cada concepto se caracteriza por dos elementos: propiedades y dimensiones (rango de variabilidad de cada propiedad).

La Figura 1, parte a) y parte b) muestra la forma como dos de los participantes utilizan y organizan su conocimiento base en una serie de acciones o pasos para resolver un sistema de dos ecuaciones con dos incógnitas. A partir del análisis de los datos de este participante y los demás, se asignan los códigos que inicialmente se denominaron, conocimiento base y organizar procedimientos.

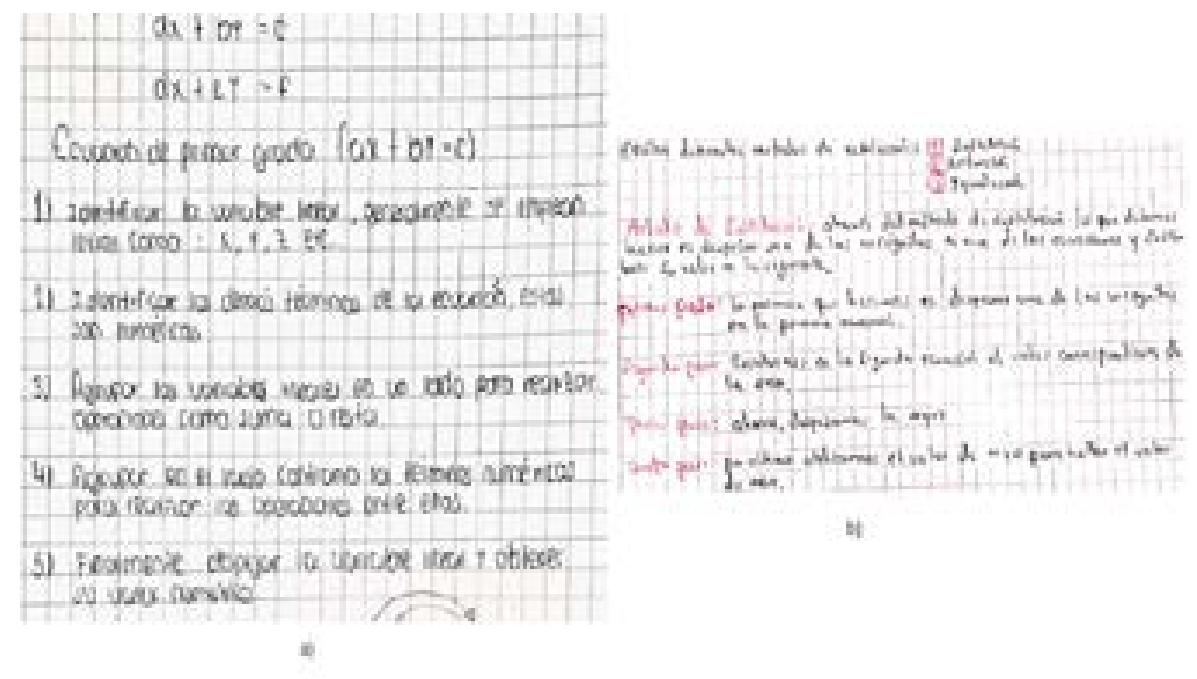

Figura 1. Conocimiento base, Organizando procedimientos 
Código 1, Conocimiento base y su forma de análisis. Concepto asignado y relacionado con la forma como los participantes utilizaron los métodos y procedimientos que tenían a su disposición en ese momento para resolver sistemas de ecuaciones lineales, sin la ayuda del profesor.

Propiedades: Procedimientos para resolver sistemas de ecuaciones lineales de 2x2 y $3 \times 3$.

Dimensiones: Métodos para resolver sistemas de ecuaciones lineales con igual número de ecuaciones y de incógnitas.
Descripción: Tipos de procedimientos utilizados para resolver sistemas de ecuaciones lineales de $n$ ecuaciones y $n$ incógnitas.

En la Figura 2, parte a) por su parte, evidencia la forma en que uno de los participantes representaba gráficamente tres ecuaciones lineales con dos variables en el plano cartesiano. La parte b) de la misma figura da muestra acerca de la forma como un participante interpreta un sistema de ecuaciones y su posible solución (a juicio de los investigadores es una forma ingenua de interpretación).

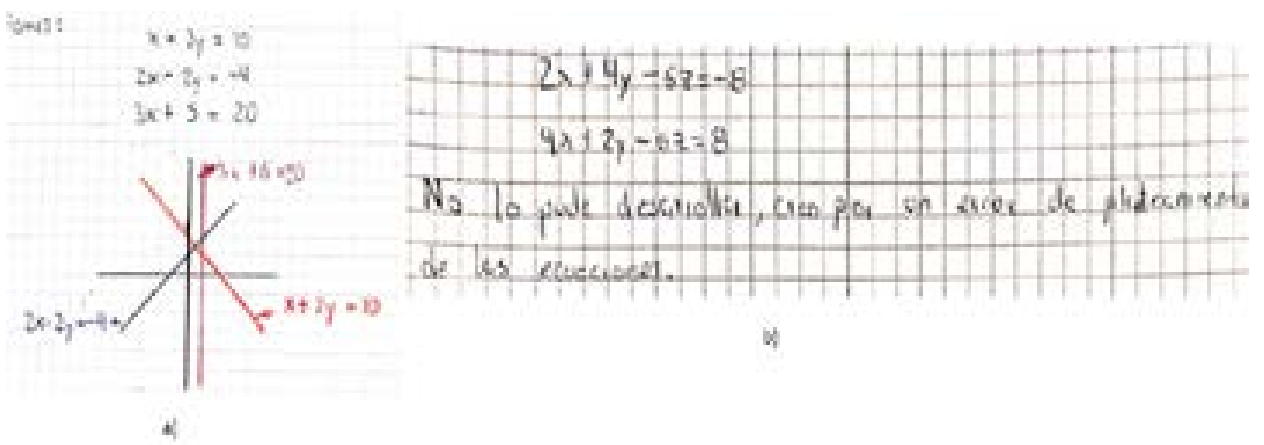

Figura 2. Representando ecuaciones e interpretando las soluciones

Código 2, Representación gráfica y su forma de análisis. Concepto asignado a la forma en que los estudiantes graficaron en dos y tres dimensiones cada una de las ecuaciones involucradas en el sistema.

Propiedades: representación gráfica de ecuaciones lineales en dos y tres variables

Dimensiones: técnicas y habilidades para representar gráficamente en el plano y en el espacio ecuaciones lineales

Descripción: estrategias para representar gráficamente en el plano y en el espacio ecuaciones en dos y tres variables.

Del proceso de codificación abierta surgieron una variedad de códigos que se fueron refinando y agrupando. La Tabla 1, muestra un resumen los primeros códigos. 
Tabla 1. Códigos iniciales tentativos en la resolución de problemas matemáticos

\begin{tabular}{|c|c|c|c|}
\hline codigo & Propledades & Dimensiones & Descripción \\
\hline $\begin{array}{l}\text { Conccimiento } \\
\text { base }\end{array}$ & $\begin{array}{l}\text { Procedimientos para } \\
\text { resclver sistemas de } \\
\text { ecuaciones lineales de } \\
2 \times 2 \text { y } 3 \times 3\end{array}$ & $\begin{array}{l}\text { Métodos para resolver } \\
\text { sistemas de ncuaciones } \\
\text { lineales de igual número de } \\
\text { ecuaciones y de incógnitas }\end{array}$ & $\begin{array}{l}\text { Tipos de procedimientos } \\
\text { utlizados para tesclvert } \\
\text { sistemas de ecuaciones } \\
\text { lineales de } 2 \times 2 \text { y } 3 \times 3\end{array}$ \\
\hline $\begin{array}{l}\text { Representación } \\
\text { gráfica }\end{array}$ & $\begin{array}{l}\text { Representacion grafica de } \\
\text { ecuaciones linoolos on } \\
\text { dosy tres variables }\end{array}$ & $\begin{array}{l}\text { Técnicas y habilidades para } \\
\text { representar gráficamente en } \\
\text { el plano y en el espacio } \\
\text { ecuacionvs linvales }\end{array}$ & $\begin{array}{l}\text { Estrategias para } \\
\text { representar graficamente } \\
\text { on ol plano y on el osescio } \\
\text { ecuaciones on dos y tres } \\
\text { variables. }\end{array}$ \\
\hline $\begin{array}{l}\text { Interpretación de } \\
\text { la solución }\end{array}$ & $\begin{array}{l}\text { Formas de interpretar la } \\
\text { solución analitica y la } \\
\text { represontación grdéca do } \\
\text { un sisterra de ecuaciones } \\
\text { lineales de } 2 \times 2,3 \times 3\end{array}$ & $\begin{array}{l}\text { Maneras de interpretar la } \\
\text { solución Única infinitas } \\
\text { soluciones y no solueion al } \\
\text { sistema de esuaciones de } \\
2 \times 2,3 \times 3\end{array}$ & $\begin{array}{l}\text { Formas de interpretar los } \\
\text { tipos de soluciones a un } \\
\text { sistema de ecuaciones } \\
\text { lineales de } 2 \times 2,3 \times 3 \text {. }\end{array}$ \\
\hline $\begin{array}{l}\text { Organizando } \\
\text { procedimientos }\end{array}$ & $\begin{array}{l}\text { Pasos o acciones } \\
\text { secuenciales para resolver } \\
\text { un sisterua de } 2 \times 2\end{array}$ & $\begin{array}{l}\text { Serie de pasos o acciones, } \\
\text { siguiendo un orden para } \\
\text { resolver sistemas de } \\
\text { ecuaciones de } 2 \times 2\end{array}$ & $\begin{array}{l}\text { Formas do organizar y } \\
\text { recrganizar pascs o } \\
\text { acciones para rescher un } \\
\text { sistema de ecuaciones } \\
\text { lineales de } 2 \times 2\end{array}$ \\
\hline
\end{tabular}

El análisis de datos continua con los procesos de codificación axial y selectiva. La tarea de comparar y contrastar los códigos tentativos condujo a las primeras categorías. Finalmente, el investigador se aleja de los datos con el propósito de analizar y buscar conceptos más abstractos que generalicen y describan los datos. La Figura 3 y la Figura 4, evidencian el trabajo realizado por los participantes codificados como E4 SA2, E15 SA2. Donde E4 significa estudiante 4 , mientras que SA2 representa la secuencia de aprendizaje 2 .
En la Figura 3, partes a) y b) muestra el trabajo del estudiante E4, cuando utiliza la representación matricial de la forma $[A \vdots b]$ correspondiente al sistema de ecuaciones lineales dado. La forma en que utiliza las operaciones elementales por renglón (por filas), para llevar la matriz $[A \vdots b]$ a una matriz equivalente de la forma escalonada. Este trabajo condujo al estudiante a interpretar y entender que el sistema de ecuaciones dado tiene infinitas soluciones, con tres variables libres a las cuales asigna números reales. De igual manera se evidencia como el participante utiliza la representación matricial $A$. $x=b$ como estrategia para verificar sus resultados. 


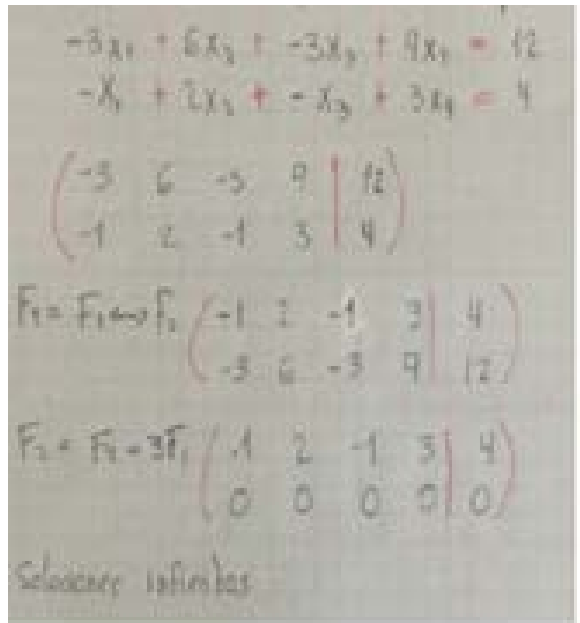

$x^{3}$

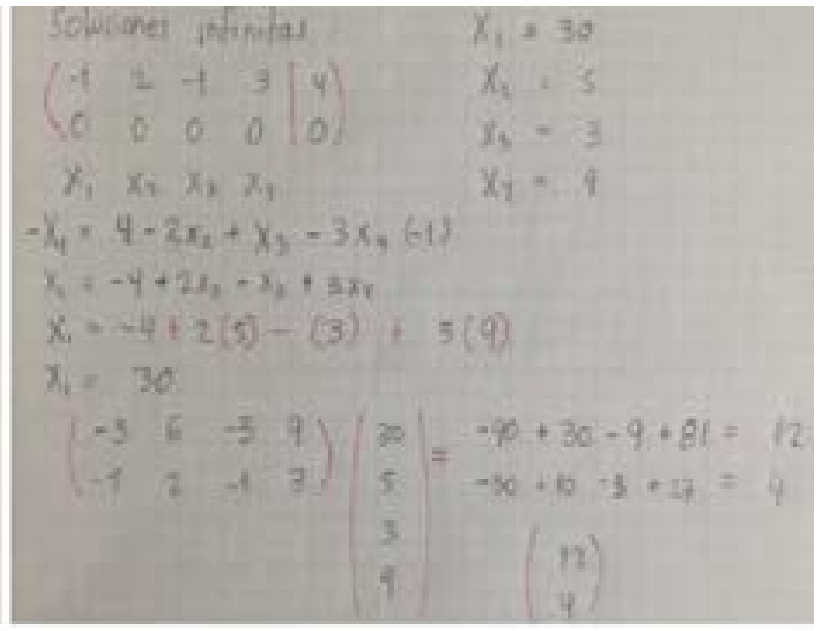

21

Figura 3. Acciones E5 SA2

La Figura 4, partes a) y b) por su parte, muestra como el participante codificado como E15, primero utiliza representación matricial $\left[A: I_{n}\right]$ como acción inicial que le permite hallar la inversa de la matriz $\mathrm{A}$, colocando al lado la matriz idéntica de orden n. La Figura 4 parte b), muestra como el estudiante utiliza letras y símbolos para representar las operaciones elementales por renglón y a la vez hace un trabajo auxiliar de estas operaciones correspondiente a estos cálculos, que generalmente es obviado en los libros de texto.

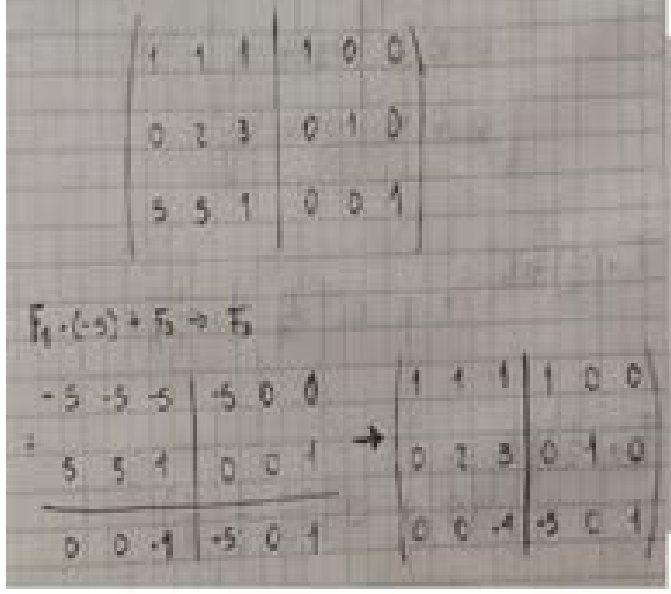

๑)

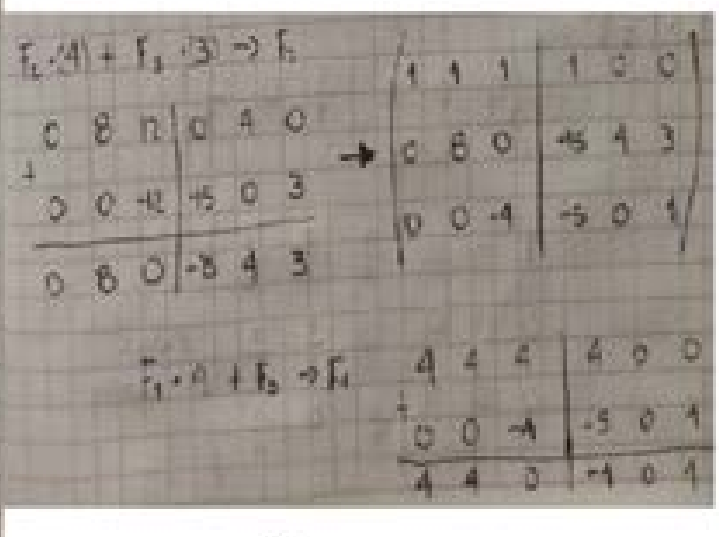

b)

Figura 4. Acciones E15 SA2

El análisis de las manifestaciones estos dos participantes y sus compañeros dio origen a una de las dos categorías centrales, que se denominó, estrategias en resolución de sistemas de ecuaciones lineales, que a su vez la conforman las subcategorías conocimiento base, formalizando, generalizando y probando inductivamente. La Tabla 2, muestra esta categoría junto a sus categorías y su descripción. 
Tabla 2. Estrategias en la resolución de sistemas de ecuaciones lineales

\begin{tabular}{|c|c|c|}
\hline \multicolumn{3}{|c|}{ Categorla central 1: estrategios en la resolución do sistemas do ecuaciones lineales } \\
\hline Categorias & Subeategorias & Descripcion \\
\hline $\begin{array}{l}\text { Conocimiento } \\
\text { bose }\end{array}$ & $\begin{array}{l}\text { Utilzando procedimientos conocidos } \\
\text { Representando graficamente } \\
\text { Interprecando soluciones }\end{array}$ & $\begin{array}{l}\text { Formas de resclver sisternas de ecuaciones } \\
\text { lineales de } 2 \times 2,3 \times 3, \text { reprosentar o interprotar } \\
\text { soluciones }\end{array}$ \\
\hline Formalizando & $\begin{array}{l}\text { Asignando letras, signos, simbolos } \\
\text { Representando operaciones } \\
\text { elementales por rengion }\end{array}$ & $\begin{array}{l}\text { Formas de asignar lotras, signos y simbolos para } \\
\text { representar sistemas de ecuaciones y } \\
\text { operaciones olomentales por renglón }\end{array}$ \\
\hline Generalizando & $\begin{array}{l}\text { Métodos para resolver sistemas de m } \\
\text { ecuaciones lineales con n inedgnitas } \\
\text { Organizando y reorganizando pasos } \\
\text { o acciones } \\
\text { Tipos de soluciones }\end{array}$ & $\begin{array}{l}\text { Formas de ir de lo particular a lo general en } \\
\text { métodos y procedimientos para solucionar } \\
\text { sistemas de } m \text { ecuaciones lineales con } n \\
\text { inégnitas y sustipos de solucion. }\end{array}$ \\
\hline $\begin{array}{l}\text { Probando } \\
\text { inductivamente }\end{array}$ & $\begin{array}{l}\text { Ejemplos, contraejemplos } \\
\text { Explicando causas y razones }\end{array}$ & $\begin{array}{l}\text { Maneras de uclizar ejemplos, contraejemplos para } \\
\text { convencerse a si mismo y convencer a los demas. }\end{array}$ \\
\hline
\end{tabular}

La resolución de problemas de física. En la etapa 2 del trabajo, se implementan las tres secuencias de aprendizaje finales. En la SA4, cuestión 1, se propuso a los participantes la tarea de interpretar y entender un problema de un circuito eléctrico, resuelto en el libro clásico de Algebra Lineal de Koman y Hill (2006). Los estudiantes tenían que descubrir, interpretar y escribir una serie de pasos que posiblemente siguieron los autores para resolver el problema. Debían también complementar algunas acciones (triviales para los autores, no para el estudiante) que no aparecen explicitas en la solución, además de explicar y justificar desde la matemática y la física todo el procedimiento.

Más adelante, en la cuestión 2 de la SA5, los estudiantes tenían que resolver el mismo problema, siguiendo los pasos y acciones que generaron en SA4, con una variante: ahora la corriente fluye en sentido contrario al problema propuesto en el libro. Luego de resolver el problema tenían que buscar diferencias y similitudes entre los procesos de resolución y los resultados hallados.
Al final de la secuencia de aprendizaje 5 (SA5), se pidió que volvieran a revisar los pasos para resolver problemas que habían elaborado y que escribieran ahora otra serie de acciones más generales para resolver este tipo de problemas. Ante esta cuestión el participante E7, escribió los siguientes pasos o acciones (se transcribe tal y como lo hizo el estudiante):

E7: Antes de empezar a resolver el circuito se deben analizar las mallas, debemos saber que baterías, resistencias, intensidades, nodos, cuantas mallas simples y compuestas tenemos, entre otros. Este análisis se debe hacer para saber que tenemos que buscar. 1) Asignamos direcciones arbitrarias a estas corrientes (si la dirección fluye en sentido o al contrario de las manecillas del reloj. Esto se hará en los diagramas o circuitos

Nota: de aquí en adelante los pasos pueden variar según lo que se necesita encontrar (baterías, resistencias $\mathrm{o}$ intensidades). 2) 
Para resolver estos ejercicios la idea es recorres las mallas simples y/o compuestas para convertir lo que obtenemos en ecuaciones y poder resolverlo. 3) Después de obtener las ecuaciones es necesario resolverlas para esto yo usaré wxMaxima. 4) Los valores que obtengamos será el valor de las incógnitas.

\begin{abstract}
Pasos especiales: como tal no son los pasos para resolver, pero sirven para comprobar que quedó bien resuelto. 1) comprobamos que los valores obtenidos son los de las ecuaciones para esto yo usaré la multiplicación de matrices, 2) comprobamos reemplazando lo valores obtenidos en el circuito usando las leyes de Kirchhoff
\end{abstract}

Mientras que la Figura 5, en sus partes a), b) y c) evidencia el trabajo manifestado por el participante, codificado como E13.

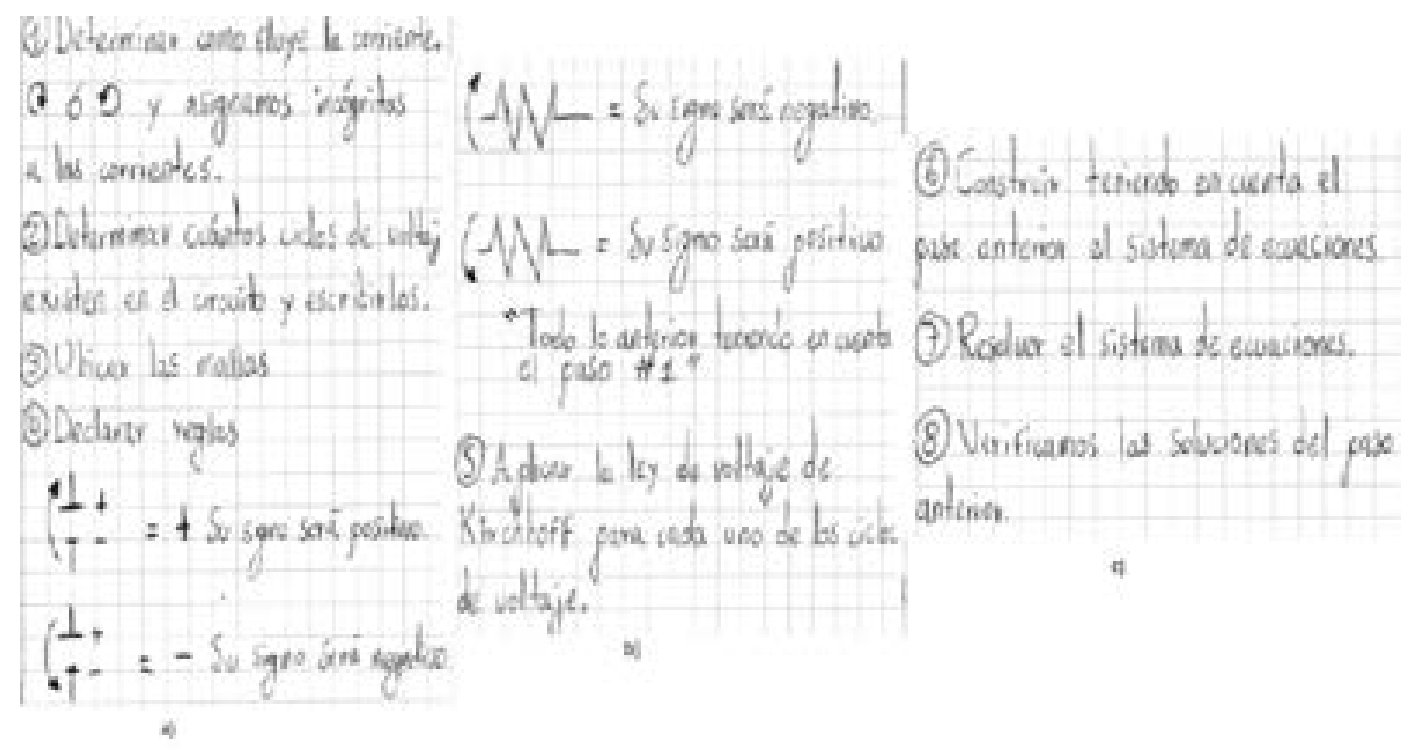

Figura 5. Pasos o acciones E13 SA5

Tanto el estudiante E7, como el de E13, proponen pasos o acciones (a su manera) y la forma cada uno de ellos hace uso de su lenguaje natural para organizar y reorganizar su conocimiento en una estrategia compuesta por una serie de acciones como proceso para resolver problemas que involucran circuitos. En contraste, también se presentaron casos de algunos estudiantes que se les dificultó elaborar y construir estas acciones. El buscar respuestas de la razón de estas dificultades no estuvo al alcance del presente trabajo.
La Figura 6, por su parte muestra como el participante E17 en la secuencia de aprendizaje 6 (E17 SA6) puso en juego los pasos o acciones que elaboró en su trabajo anterior, para resolver un problema más complejo que el resuelto en el libro de texto. En las partes a), b) y c) de la misma figura, se observa como el estudiante, primero identifica información conocida y desconocida y utiliza letras, signos y símbolos para representar esta información. Mientras que en las partes d), e), f), g) y h) utiliza conocimientos de física (leyes 
de Kirchhoff) en nodos, mallas y los representa matemáticamente mediante ecuaciones, siguiendo el sentido dado al flujo de corriente.

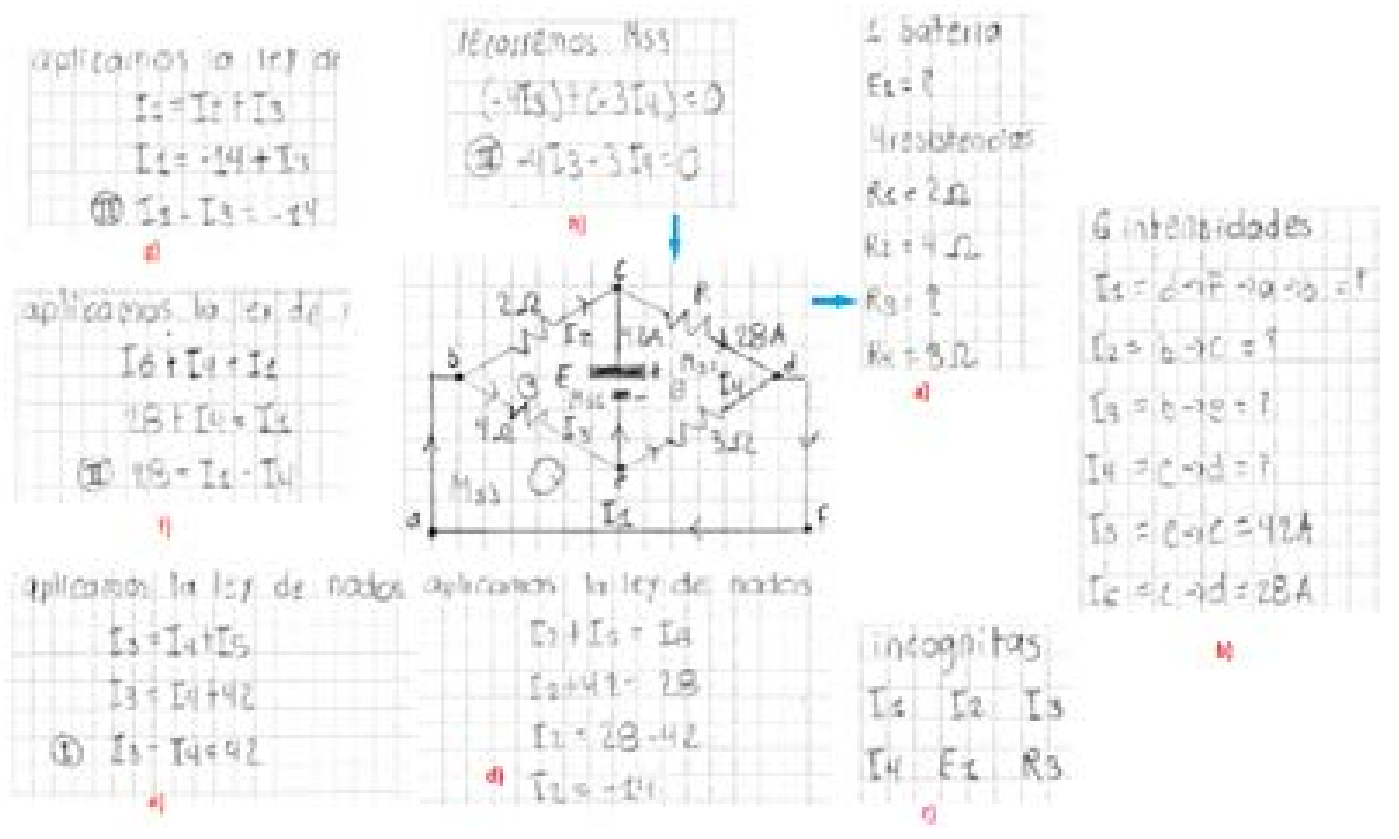

Figura 6. Acciones para resolver problemas E17 SA6

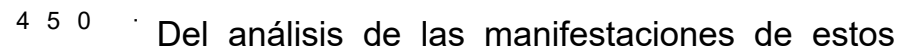
participantes y los demás seleccionados como incidentes, surgieron los primeros códigos, que se denominaron, reconocer, suponer y asignar.

Código reconocer. Hace referencia a la manera como cada participante identifica los elementos principales en la representación gráfica del circuito.

Propiedades: información conocida y desconocida en el circuito

Dimensiones: elementos que estructuran el problema como nodos, mallas, resistencias, baterías.

Descripción: formas de interpretar e identificar los tipos de elementos que estructuran el problema.
Código suponer: se refiere a manera en que los participan asumen el sentido del flujo de corriente.

Propiedades: Sentido dado al concepto físico flujo de corriente.

Dimensiones: Tipo asignación al flujo de corriente, ya sea en sentido de las manecillas del reloj o en sentido contrario.

Descripción: Formas de suponer el flujo de corriente, esto le va a permitir utilizar leyes físicas para construir el sistema de ecuaciones.

El trabajo de análisis y codificación continua, la Tabla 3 muestra los códigos y categorías iniciales en el proceso de codificación abierta y axial. 
Tabla 3. Códigos iniciales resolución problemas de física

\begin{tabular}{|c|c|c|c|}
\hline Codigo & Propledades & Dimensiones & Descripción \\
\hline Rec & $\begin{array}{l}\text { Intormación conocida y } \\
\text { desconocida en el circuito }\end{array}$ & $\begin{array}{l}\text { Elementos que estructuran el } \\
\text { problema como nodos. } \\
\text { mallas. resistencias, } \\
\text { baterias. }\end{array}$ & $\begin{array}{l}\text { Formas de interpretar e } \\
\text { identifcar los tipos de } \\
\text { elementos que estructuran } \\
\text { ol problema. }\end{array}$ \\
\hline Suponer & $\begin{array}{l}\text { Sentido dado al concepto fisico } \\
\text { fujo de corriente }\end{array}$ & $\begin{array}{l}\text { Tipo asignación al flujo de } \\
\text { corrionte, ya sea on sentido } \\
\text { de las manecillas del reloj o } \\
\text { viceversa }\end{array}$ & $\begin{array}{l}\text { Formas de suponer el fujo } \\
\text { de corriente, esto le va a } \\
\text { permitir utiluar leyes } \\
\text { fisicas para construif el } \\
\text { sistema de ecuaciones }\end{array}$ \\
\hline Asign & $\begin{array}{l}\text { Utizar letras, signos y } \\
\text { simbolos para representar } \\
\text { conceptos y leyes fisicas }\end{array}$ & $\begin{array}{l}\text { Tipos letras, signos y } \\
\text { simbolos utilizados para } \\
\text { representar conceptos } \\
\text { fisicos }\end{array}$ & $\begin{array}{l}\text { Formas de asignar letras, } \\
\text { signos y simbolos en la } \\
\text { representacion de } \\
\text { conceptos y leyes fisicas }\end{array}$ \\
\hline Formular & $\begin{array}{l}\text { hes matemabicas en } \\
\text { e ecuaciones lineales } \\
\text { resentan conceptos. } \\
\text { is y leyes fisicas }\end{array}$ & $\begin{array}{l}\text { Tipo de ecuaciones lineales } \\
\text { asignadas para representar } \\
\text { conceptos y relaciones } \\
\text { fisicas }\end{array}$ & $\begin{array}{l}\text { Sistemo de ecuaciones } \\
\text { lineales que representan } \\
\text { conceptos relaciones y } \\
\text { leyes de Kirchhoff }\end{array}$ \\
\hline $\begin{array}{l}\text { Opera } \\
\text { por ren }\end{array}$ & $\begin{array}{l}\text { Operaciones artméticas entre } \\
\text { y en renglones utilizadas para } \\
\text { resolver sistemas de } \\
\text { ecuaciones lineales }\end{array}$ & $\begin{array}{l}\text { Tipo de operaciones } \\
\text { elementales por renglón } \\
\text { permidas }\end{array}$ & $\begin{array}{l}\text { Formas de representar y } \\
\text { utilizar las operaciones } \\
\text { elementales por renglon } \\
\text { para resolver sistemss de } \\
\text { ecuaciones lineales }\end{array}$ \\
\hline Verifeacion & $\begin{array}{l}\text { Operaciones aritméticas para } \\
\text { verificar la veracidad de la } \\
\text { solución del sistema de } \\
\text { ecuaciones }\end{array}$ & $\begin{array}{l}\text { Formas de verificar los } \\
\text { resultados luego de haber } \\
\text { resuelto ol sistecns de } \\
\text { ecuaciones lineales }\end{array}$ & $\begin{array}{l}\text { Formas de verifcar la } \\
\text { solución (es) al sistema de } \\
\text { ecuaciones lineales que } \\
\text { representa el circuito }\end{array}$ \\
\hline
\end{tabular}

El proceso de codificación selectiva inicia dando origen a la segunda categoría central que se denominó, estrategias en la resolución de problemas de circuitos. La Tabla 4, muestra esta categoría junto a sus subcategorías. 
Tabla 4. Estrategias en la resolución de problemas de circuitos

\begin{tabular}{|c|c|c|}
\hline categonas & subcategorias & Deseripeion \\
\hline \multirow{3}{*}{ Entender } & $\begin{array}{l}\text { Reconocer informacion conccida (baterias. } \\
\text { resistencias, corrientes) y desconocida en ei circuro }\end{array}$ & \multirow{3}{*}{ 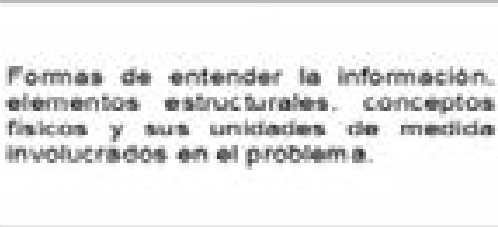 } \\
\hline & $\begin{array}{l}\text { Interpretar elementos estructurales del problema } \\
\text { como mallas. nodos. elc. }\end{array}$ & \\
\hline & $\begin{array}{l}\text { Interpretar conseptes fisices come: intensidad de } \\
\text { corriente. diferencia de potersial. voltaje y sus } \\
\text { canticiasies fisicas para cuantificarias }\end{array}$ & \\
\hline \multirow{3}{*}{ Formalizer } & $\begin{array}{l}\text { Euponer sentide en ave fure al eeneeple fuje de } \\
\text { corriente }\end{array}$ & \multirow{3}{*}{$\begin{array}{l}\text { Formas de representar et peoblema } \\
\text { fisies on tormines matemasicos } \\
\text { mediante sistemas de ecuaciones } \\
\text { lineales. }\end{array}$} \\
\hline & $\begin{array}{l}\text { Representar cenceptos, leyes fisicas y relsciones } \\
\text { mediante letras, aignos y simbolos. }\end{array}$ & \\
\hline & $\begin{array}{l}\text { Formular sistemas de ecuaciones tineales que } \\
\text { representan o modelan el problema. }\end{array}$ & \\
\hline Resolver & 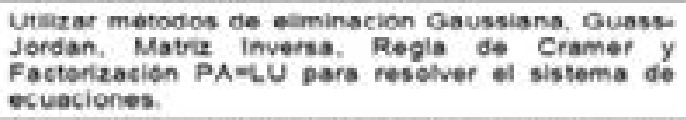 & 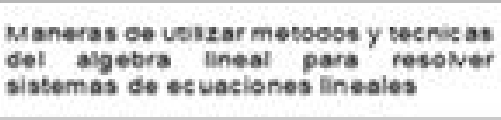 \\
\hline \multirow{3}{*}{ Probar } & 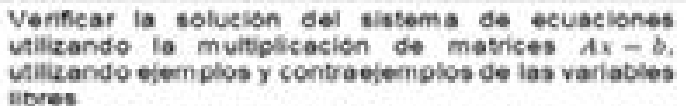 & \multirow{3}{*}{$\begin{array}{l}\text { Formas de vorificar y refexionar } \\
\text { matematica y fisicamente ecerce de } \\
\text { los tipos de solucionies halladas }\end{array}$} \\
\hline & $\begin{array}{l}\text { Determinar si las soluciones halladas cumplen las } \\
\text { exigencias deil problema planteado. }\end{array}$ & \\
\hline & $\begin{array}{l}\text { Refexionar acerca de los tipos de solucion } \\
\text { metematice haliada y su significado en el problema } \\
\text { real }\end{array}$ & \\
\hline \multirow[b]{2}{*}{ Extender } & $\begin{array}{l}\text { Formas de organizar y reorgenizar pasos y acciones } \\
\text { para resolver problemas ave involucran circuitos. }\end{array}$ & \multirow{2}{*}{$\begin{array}{l}\text { Maneras de erganizar y reerganizar af } \\
\text { conocimiento pare resolver problem as } \\
\text { de circuitos electricos y uilizarlo para } \\
\text { resolver probiemas que involueran } \\
\text { otro tipo de cirsultes. }\end{array}$} \\
\hline & $\begin{array}{l}\text { Maneras de velizar los conecimientes en la } \\
\text { resolucion de problemes de circultos electrices a } \\
\text { otrus tipos de circultos corno fujo de agua por un } \\
\text { acueducto o flujo de autos en una cludad. }\end{array}$ & \\
\hline
\end{tabular}

\section{RESULTADOS}

Como resultado de los procesos de codificación abierta, axial, selectiva, el muestreo teórico y saturación teórica (cuando los datos nuevos, no hacen aportes significativos a las categorías construidas) surgieron dos categorías centrales interdependientes como procesos, que se denominaron: estrategias para resolver problemas de física (RPF) y estrategias para resolver problemas de matemáticas (RPM). La Figura 7, muestra un esquema de estas categorías en un flujo de trabajo dinámico y cíclico.

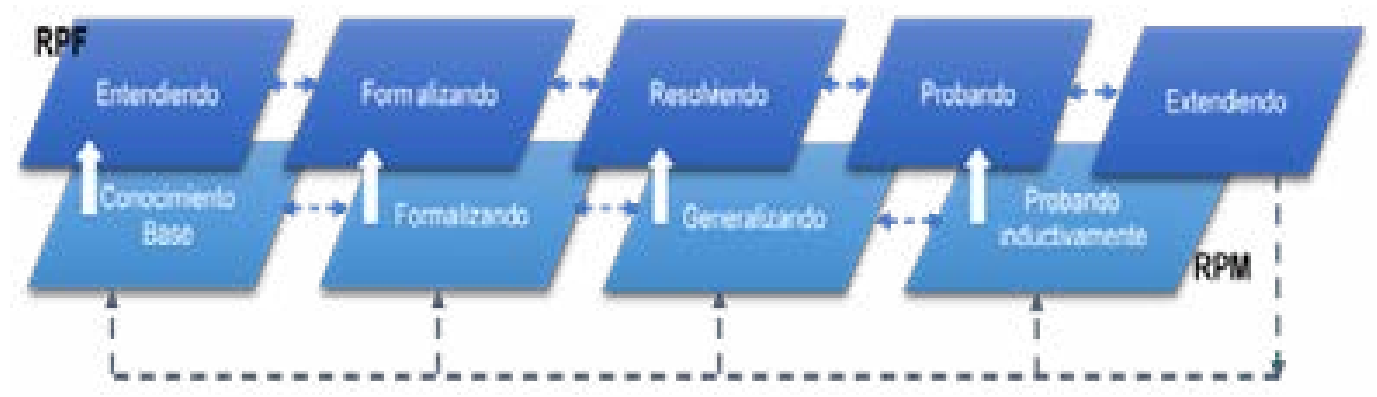

Figura 7. Categorías centrales como procesos 
La Figura 7, muestra un proceso continuo, donde los participantes realizaron un trabajo permanente de ida y vuelta sobre el problema, con trabajo simultaneo cuando resuelven problemas de circuitos que involucran sistemas de ecuaciones lineales y sus métodos de solución. En el esquema cíclico se destaca que: luego de que los participantes resuelven un problema su conocimiento base se incrementa y éste nuevo conocimiento es útil para resolver nuevos problemas. Por ejemplo, primero resolvieron problemas de circuitos eléctricos y luego utilizaron esas estrategias para resolver problemas de agua fluye por un acueducto y la cantidad de vehículos por las calles de una ciudad.

Cada uno de estos procesos se caracteriza por: a) ser variables, puesto que cada participante los realiza a su manera, siguiendo sus conocimientos, b) cada categoría está compuesta por subcategorías, c) se puede conceptualizar de diferentes maneras y d) hay una acción e interacción entre las categorías que constituyen cada proceso y a la vez hay interacción entre las dos categorías centrales.

\section{CONCLUSIONES Y DISCUSIÓN}

El trabajo de los participantes, la estrategia de investigación propuesta, junto a los procesos de codificación y análisis de datos, posibilitó la construcción inductiva de dos categorías centrales. La primera se denominó estrategias para resolver problemas de física (RPF), compuesta por un flujo de acciones e interacciones entre las subcategorías entendiendo, formalizando, resolviendo, probando y extendiendo. Estas subcategorías coinciden con las últimas cinco etapas propuestas por Gaigher, Rogan y Braun (2007) y los procesos de formalización y resolucón del problema de Jensen, Niss y Jankvist (2017).

La segunda categoría conceptualizada como estrategias para resolver problemas de matemáticas (RPM), muestra un flujo de trabajo entre las subcategorías conocimiento base, formalizando, generalizando y probando inductivamente. Estas subcategorías están inmersas en las cuatro etapas propuestas por Polya (1945), las fases de entrada, ataque y revisión de Mason, Burton y Stacey (2010), las dimensiones cognitiva y heurística de Schoenfeld (2016) y los procesos de representación y solución del problema Mayer (2010). Las dos categorías centrales se encuentran inmersas en el modelo propuesto por Greeno (1989).

En lo que respecta a las relaciones entre los procesos simultáneos de resolución de problemas de física y resolución de problemas de matemática, cabe destacar que:

$\checkmark$ Al momento de resolver problemas matemáticos que involucran sistemas de ecuaciones lineales los estudiantes utilizan diferentes métodos y procedimientos para resolver los sistemas de ecuaciones, manipulando expresiones algebraicas con sentido matemático representado por las relaciones entre los pasos o acciones de cada uno de los métodos (como técnica), pero sin ningún sentido en el contexto del problema físico.

$\checkmark$ Las leyes físicas, como las leyes de Kirchhoff requieren y originan expresiones algebraicas o fórmulas matemáticas (ecuaciones) para ser representadas.

$\checkmark$ Al momento de verificar soluciones al problema, las soluciones al sistema de ecuaciones lineales pueden estar bien, pero si el sistema de ecuaciones no representa bien el problema, las soluciones no corresponden al problema planteado. Es decir, existe una relación directa entre el problema bien formalizado 
matemáticamente y el problema físico real.

$\checkmark$ Los errores en los métodos y técnicas para resolver sistemas de ecuaciones lineales conducen a soluciones erróneas del problema propuesto, pero inducen al solucionador en un proceso verificación, análisis y reflexión.

$\checkmark \quad$ La forma de interpretación (ingenuas, erróneas, acertadas) del solucionador a las respuestas al sistema de ecuaciones lo conducen a formas de entender correctas o incorrectas.

$\checkmark \quad$ La resolución de problemas de física puede ayudar al estudiante en los procesos de formalización del problema, así como fortalecer procedimientos y técnicas para resolver sistemas de ecuaciones.

$\checkmark$ Reconocer la relación entre el proceso de formalización o matematización del problema y la situación o contexto del problema es difícil y crucial para el estudiante.

Finalmente, los hallazgos desde este estudio de caso evidencian una relación clara de inclusión de las estrategias para resolver problemas de matemáticas entre las estrategias para resolver problemas de física, aunque el flujo de acción e interacción entre estas estrategias es simultaneo y permanente. Por tanto, las estrategias para resolver problemas de matemáticas son fundamentales y necesarias para resolver problemas de física. Se deriva de aquí la pregunta: ¿será que esta relación de inclusión es valida en la resolución de todo problema de física?

\section{REFERENCIAS BIBLIOGRÁFICAS}

Al-Omari, W., \& Miqdadi, R. (2014). The Epistemological Perceptions of the Relationship between Physics and Mathematics and Its Effect on Problem-Solving among Pre-Service Teachers at Yarmouk University in Jordan. International Education Studies, 7(5), 39-48.

Bing, T., \& Redish, E. (2009). Analyzing problem solving using math in physics: Epistemological framing via warrants. Physical Review Special Topics-Physics Education Research, 5(2), 020108. doi:10.1103/PhysRevSTPER.5.020108

Charmaz, K. (2006). Constructing grounded theory: A practical guide through qualitative analysis. London: Sage Publications.

Charmaz, K. (2014). Constructing grounded theory (2 ed.). Thousand Oaks, CA: Sage.

Corbin, J., \& Strauss, A. (2017). Conceptos básicos de la investigación cualitativa: técnicas y procedimientos para desarrollar la teoría fundamentada (4 ed.). Thousand Oaks, California, United States of America: SAGE Publications.

De Ataíde, A., \& Greca, I. (2012). Epistemic Views of the Relationship Between Physics and Mathematics: Its Influence on the Approach of Undergraduate Students to Problem Solving. Science and Education, 22(6), 1405-1421. doi: 10.1007/s11191-012-9492-2

Ernest, P. (2005). Social Constructivism and the Psychology. En P. Ernest (Ed.), Constructing Mathematical Knowledge: Epistemology and Mathematics. London: Taylor \& Francis e-Library. 
Gaigher, E., Rogan, J., \& Braun, M. (2007). Exploring the Development of Conceptual Understanding through Structured Problem-solving in Physics. International Journal of Science Education, 29(9), 1089-1110. doi:10.1080/09500690600930972

Greeno, J. (1989). Situations, mental models, and generative knowledge. En D. Klarh, \& K. Kotovsky (Edits.), Complex information processing: The impact of Herbert A. Simon (págs. 285-318).

Jensen, J., Niss, M., \& Jankvist, U. (2017). Problem solving in the borderland between mathematics and physics. International Journal of Mathematical Education in Science and Technology, 48(1), 1-15. doi: $10.1080 / 002073$ 9X.2016.1206979

Koman, B., \& Hill, D. (2006). Algebra Liineal (8 ed.). (V. Ibarra Mercado, Trad.) México, México: Pearson Educación.

Mason, J., Burton, L., \& Stacey, K. (2010). Thinking Mathematically (2 ed.). Harlow, UK: Pearson Education Limited.

Mayer, R. (2010). Problem Solving and Reasoning. International Encyclopedia of Education, 273-278. doi:10.1016/ B978-0-08-044894-7.00487-5

Miles, M., Huberman, A., \& Saldaña, J. (2018). Qualitative data analysis: $A$ methods sourcebook (3 ed.). USA: Sage publications.

Polya, G. (1945). How To Solve It. Princeton: Princeton University Press.

Polya, G. (1981). Mathematical Discovery. New York: Wiley.

Quale , A. (2010). On the Role of Mathematics in Physics. Science \& Education, 20(34), 359-372. doi:10.1007/s11191-0109278-3

Redish, E. (2006). Problem solving and the use of math in physics courses. arXiv:physics/0608268v1.

Redish, E., \& Bing, T. (2009). Using math in physics: Warrants and epistemological frames. Girep-Epec \& Phec. 71.

Scanlon, E. (1993). Solving the problem of physics problem solving. International Journal of Mathematical Education in Science and Technology, 24(3), 349358. doi:10.1080/0020739930240303

Schoenfeld, A. H. (2016). Learning to think mathematically: Problem solving, metacognition, and sense making in mathematics (Reprint). Journal of Education, 196(2), 1- 38. doi:10.1177/002205741619600202

Von Glasersfeld, E. (2013). Radical constructivism (Vol. 6). Routledge. 